\title{
On the existence of weak solutions to the Cauchy problem for a class of quasilinear hyperbolic equations with a source term
}

\author{
Filipa CAETANO
}

\author{
Dep. Matemática, \\ Universidade Nova de Lisboa and CMAF/UL, \\ Faculdade de Ciências e Tecnologia, \\ Quinta da Torre, Monte da Caparica, \\ 2829-516 Caparica, PORTUGAL \\ fmc@fct.unl.pt
}

Recibido: 10 de Enero de 2002

Aceptado: 2 de Octubre de 2003

\section{ABSTRACT}

Following the ideas of D. Serre and J. Shearer in [16], we prove in this paper the existence of a weak solution of the Cauchy problem for the second order quasilinear hyperbolic equation

$$
\phi_{t t}-\sigma^{\prime}\left(\phi_{x}\right) \phi_{x x}+F(\phi)=0, \quad(x, t) \in \mathbb{R} \times[0,+\infty[,
$$

where $F(\phi)$ is a suitable source term.

Key words: Quasilinear hyperbolic equations, conservation laws, viscosity approximation, compensated compactness, Young measures

2000 Mathematics Subject Classification: Primary: 35L70; Secondary: 35L45

\section{Introduction and main results.}

This paper presents a study of the initial values problem for the second order quasilinear equation

$$
\phi_{t t}-\sigma^{\prime}\left(\phi_{x}\right) \phi_{x x}+F(\phi)=0, \quad(x, t) \in \mathbb{R} \times[0,+\infty[,
$$

This research has been partially supported by FCT, FEDER and the ICCTI/CNRS program. 
following the work of J. P. Dias and M. Figueira, who studied this problem in [4], considering particular $F$ and $\sigma$, namely

$$
F(\phi)=\phi^{3} \text { and } \sigma(u)=u+\frac{u^{3}}{3} .
$$

Previously, P. Marcati and R. Natalini proved in [9] a result of existence of a Lipschitz continuous solution to the Cauchy problem for equation (1) with bounded, compactly supported initial data, in the $L^{\infty}$ framework, by using an approximating scheme of Lax-Friedrichs kind, and imposing some restrictions on $F$, namely $F(0)=0$ and $F^{\prime}$ bounded.

Here, we generalize these authors' work and we prove the existence of weak solution for equation (1), with initial data

$$
\phi(x, 0)=\phi_{0}(x) \in H^{3}(\mathbb{R}), \quad \phi_{t}(x, 0)=\phi_{1}(x) \in H^{2}(\mathbb{R}) .
$$

To this purpose, we follow the method of D. Serre and J. Shearer ([16]), who proved, by using the compensated compactness method developed by F. Murat, L. Tartar and R. DiPerna ([11], [18], [5]) and $L^{\eta}$ Young measures, the existence of weak solution to the Cauchy problem for the hyperbolic system of conservation laws

$$
\left\{\begin{array}{l}
u_{t}-v_{x}=0, \\
v_{t}-\sigma^{\prime}(u) u_{x}=0 .
\end{array}\right.
$$

We consider $F: \mathbb{R} \longrightarrow \mathbb{R}$ a smooth function such that $F(0)=0, F^{\prime}(\phi) \geq 0$, $\forall \phi \in \mathbb{R}$, and $|F(\phi)| \leq c_{1}\left|\phi^{p}\right|$, for some $c_{1}>0, p \geq 1$. We put $G(\phi)=\int_{0}^{\phi} F(\theta) d \theta$.

The function $\sigma: \mathbb{R} \longrightarrow \mathbb{R}$ is in the same conditions of [16], a smooth function such that $\sigma(0)=0$ and satisfying the following hypotheses:

$$
\begin{aligned}
& \text { H1 } \exists c>0: \sigma^{\prime}(u) \geq c, \forall u \in \mathbb{R} ; \\
& \text { H2 } \sigma^{\prime \prime}(\lambda) \neq 0, \forall \lambda \in \mathbb{R}, \text { or } \exists \lambda_{0} \in \mathbb{R}: \sigma^{\prime \prime}\left(\lambda_{0}\right)=0, \sigma^{\prime \prime}(\lambda) \neq 0, \forall \lambda \neq \lambda_{0} ; \\
& \text { H3 } \frac{\sigma^{\prime \prime}}{\left(\sigma^{\prime}\right)^{5 / 4}}, \frac{\sigma^{\prime \prime \prime}}{\left(\sigma^{\prime}\right)^{7 / 4}} \in L^{2}(\mathbb{R}) ; \frac{\sigma^{\prime \prime}}{\left(\sigma^{\prime}\right)^{3 / 2}}, \frac{\sigma^{\prime \prime \prime}}{\left(\sigma^{\prime}\right)^{2}} \in L^{\infty}(\mathbb{R}) ;
\end{aligned}
$$

H4 We define $\Sigma(u)=\int_{0}^{u} \sigma(s) d s . \frac{\sigma(u)}{\Sigma(u)} \longrightarrow 0,|u| \rightarrow+\infty$ and there are $m$ and $q$, $q>1 / 2$, such that $\left(\sigma^{\prime}(u)\right)^{q} \leq m(1+\Sigma(u))$.

We point out that, under these hypotheses, $G(\phi) \geq 0, \forall \phi$, and $\Sigma(u) \geq c \frac{u^{2}}{2}$. It is easy to check that the functions $F$ and $\sigma$ defined by (2) satisfy all these conditions and that $\mathrm{H} 3-\mathrm{H} 4$ hold for any $\sigma$ with a suitable polynomial like behaviour. 
The Cauchy problem for equation (1) will be considered in the following equivalent formulation: we put $u=\phi_{x}, v=\phi_{t}$; then (1) reduces to the quasilinear system

$$
\left\{\begin{array}{l}
u_{t}-v_{x}=0 \\
v_{t}-\sigma^{\prime}(u) u_{x}+F(\phi)=0, \quad \phi(x, t)=\int_{0}^{t} v(x, \tau) d \tau+\phi_{0}(x) .
\end{array}\right.
$$

We consider the Cauchy problem for this system with initial data

$$
\begin{gathered}
u(\cdot, 0)=\phi_{0 x}(\cdot, 0)=u_{0}, \quad v(\cdot, 0)=\phi_{1}(\cdot, 0)=v_{0}, \\
\phi_{0} \in H^{3}(\mathbb{R}), \quad u_{0}, v_{0} \in H^{2}(\mathbb{R}) .
\end{gathered}
$$

Let

$$
E(u, v)=\int_{\mathbb{R}} \frac{v^{2}(x)}{2}+\Sigma(u(x)) d x
$$

be the energy functional and, setting $\eta(u, v)=\frac{v^{2}}{2}+\Sigma(u)$, we consider

$$
L^{\eta}=\left\{(u, v) \in\left(L_{l o c}^{1}(\mathbb{R})\right)^{2}: E(u, v)<+\infty\right\}
$$

the space of functions with finite energy. Let $L^{\infty}\left(\left[0,+\infty\left[; L^{\eta}\right)\right.\right.$ be the space of the pairs of functions $(u, v)$, defined a. e. and measurable in $[0,+\infty[\times \mathbb{R}$, such that $(u(t), v(t)) \in L^{\eta}$, a. e. $t \in[0,+\infty[$, and $\operatorname{ess} \sup E(u(t), v(t))<+\infty$.

$$
[0,+\infty[
$$

A pair of functions $(u, v) \in L^{\infty}\left(\left[0,+\infty\left[; L^{\eta}\right)\right.\right.$ is called a weak solution of the Cauchy problem (4), (5), if

$$
\begin{aligned}
\int_{\mathbb{R}} \int_{0}^{+\infty}\left(u \varphi_{t}-v \varphi_{x}\right) d x d t+\int_{\mathbb{R}} u_{0} \varphi(x, 0) d x+ \\
\int_{\mathbb{R}} \int_{0}^{+\infty}\left(v \psi_{t}-\sigma(u) \psi_{x}-F(\phi) \psi\right) d x d t+\int_{\mathbb{R}} v_{0} \psi(x, 0) d x=0,
\end{aligned}
$$

for any $\varphi, \psi \in C_{0}^{\infty}(\mathbb{R} \times[0,+\infty[)$.

A pair of functions $p, q: \mathbb{R}^{2} \longrightarrow \mathbb{R}$ is an entropy-entropy flux pair for the system (4), if all smooth solutions $(u, v)$ of (4) also satisfy

$$
p(u, v)_{t}+q(u, v)_{x}+\nabla p \cdot(0, F(\phi))=0 .
$$

It is sufficient that $p$ and $q$ satisfy

$$
\nabla p(u, v) \cdot \nabla f(u, v)=\nabla q(u, v), \quad \forall(u, v) \in \mathbb{R}^{2},
$$

where $f(u, v)=(-v,-\sigma(u))$. 
We call $(u, v)$ a weak entropy solution of $(4),(5)$, if $(u, v)$ is a weak solution that also satisfies

$$
p(u, v)_{t}+q(u, v)_{x}+\nabla p(u, v) \cdot(0, F(\phi)) \leq 0,
$$

in the sense of distributions in $\mathbb{R} \times] 0,+\infty[$, for any convex entropy $p$ of flux $q$.

We present now the main result of this work:

Theorem 1.1. We assume the above conditions for $F$ and $\sigma$. If $u_{0}$ and $v_{0}$ satisfy (6) and $\left(u_{0}, v_{0}\right) \in L^{\eta}$, then there is a global weak solution $(u, v)$ of the Cauchy problem (4), (5) in $L^{\infty}\left(\left[0,+\infty\left[; L^{\eta}\right)\right.\right.$ that satisfies the entropy inequality (9) for the entropy-entropy flux pair defined by

$$
p(u, v)=\eta(u, v)=\frac{v^{2}}{2}+\Sigma(u), \quad q(u, v)=-v \sigma(u) .
$$

To prove this result, we consider a sequence of viscosity functions $\left(u_{\varepsilon}, v_{\varepsilon}\right)$, solutions of the approximated system

$$
\left\{\begin{array}{l}
u_{\varepsilon t}-v_{\varepsilon x}=0 \\
v_{\varepsilon t}-\sigma^{\prime}\left(u_{\varepsilon}\right) u_{\varepsilon x}+F\left(\phi_{\varepsilon}\right)=\varepsilon \Delta v_{\varepsilon}, \quad \phi_{\varepsilon}(x, t)=\int_{0}^{t} v_{\varepsilon}(x, \tau) d \tau+\phi_{0}(x),
\end{array}\right.
$$

which is obtained by adding the viscosity parameter $\varepsilon \Delta \phi_{t}$ to the second member of (1).

In section 2 we prove the existence of global solution $\left(u_{\varepsilon}, v_{\varepsilon}\right)$ in $C([0,+\infty[$; $\left.H^{2}(\mathbb{R})^{2}\right) \cap C^{1}\left(\left[0,+\infty\left[; L^{2}(\mathbb{R})^{2}\right)\right.\right.$ of the Cauchy problem for system (11), with initial data

$$
u_{\varepsilon}(\cdot, 0)=\phi_{0 x}=u_{0}, \quad v_{\varepsilon}(\cdot, 0)=\phi_{1}=v_{0},
$$

In section 3 we derive energy estimates for the approximated solutions $u_{\varepsilon}$ and $v_{\varepsilon}$, which allow us to conclude that the sequence $\left(u_{\varepsilon}, v_{\varepsilon}\right)_{\varepsilon}$ is bounded in $L_{\text {loc }}^{2}(\mathbb{R} \times[0,+\infty[)$ and so we may consider a subsequence $\left(u_{\varepsilon^{\prime}}, v_{\varepsilon^{\prime}}\right)_{\varepsilon^{\prime}}$ converging weakly to $(u, v) \in$ $\left(L_{\text {loc }}^{2}\left(\mathbb{R} \times[0,+\infty[))^{2}\right.\right.$. Our aim is to prove that the pair $(u, v)$ is a global weak solution of the Cauchy problem (4), (5).

If we write the weak formulation of (11), (12),

$$
\begin{gathered}
\int_{\mathbb{R}} \int_{0}^{+\infty}\left(u_{\varepsilon} \varphi_{t}-v_{\varepsilon} \varphi_{x}\right) d x d t+\int_{\mathbb{R}} u_{0} \varphi(x, 0) d x+ \\
\int_{\mathbb{R}} \int_{0}^{+\infty}\left(v_{\varepsilon} \psi_{t}-\sigma\left(u_{\varepsilon}\right) \psi_{x}-F\left(\phi_{\varepsilon}\right) \psi\right) d x d t+\int_{\mathbb{R}} v_{0} \psi(x, 0) d x= \\
-\varepsilon \int_{\mathbb{R}} \int_{0}^{+\infty} v_{\varepsilon} \psi_{x x},
\end{gathered}
$$


we see that, if $\left(u_{\varepsilon^{\prime}}, v_{\varepsilon^{\prime}}\right) \rightarrow(u, v)$, weakly in $L_{l o c}^{2}\left(\mathbb{R} \times\left[0,+\infty[)^{2}\right.\right.$, the linear terms in the previous equation clearly converge to the correspondent terms in the equation (7). But the uniform bound in $L^{2}$ is not enough to warrant the strong local convergence of the subsequence $\left(u_{\varepsilon^{\prime}}, v_{\varepsilon^{\prime}}\right)_{\varepsilon^{\prime}}$, and the weak convergence doesn't allow us to pass to the limit the nonlinear terms $\sigma\left(u_{\varepsilon}\right)$ and $F\left(\phi_{\varepsilon}\right)$. We use the associated Young measure to represent the weak limit of the nonlinear compositions $g\left(u_{\varepsilon}, v_{\varepsilon}\right)$, of continuous functions $g$ with $\left(u_{\varepsilon}, v_{\varepsilon}\right)$. Since $L^{\infty}$ estimates are not available in this case, we follow Serre and Shearer's method ([16]), who used $L^{\eta}$ Young measures and a class of slowly growing entropy-entropy flux pairs to prove the existence of solution of the Cauchy problem for equation (3) with physical viscosity. The Young measure gives a criteria to know when the weak convergence is, in fact, strong, which happens if the measure is a Dirac mass. The theory of compensated compactness provides the compacity conditions to conclude the strong local convergence of $\left(u_{\varepsilon^{\prime}}, v_{\varepsilon^{\prime}}\right)_{\varepsilon^{\prime}}$. By applying Murat's lemma and div-curl lemma, we derive Tartar's equation. The results obtained by Serre and Shearer imply the reduction of the support of the Young measure.

\section{The approximated problem.}

In this section we consider the Cauchy problem for the approximated system (11), with initial data defined by $(12)$, where $\phi_{0} \in H^{3}(\mathbb{R}), \phi_{1} \in H^{2}(\mathbb{R})$, and $\sigma$ and $F$ as described above.

We will prove that the Cauchy problem for the nonlinear parabolic equation

$$
\phi_{t t}-\sigma^{\prime}\left(\phi_{x}\right) \phi_{x x}+F(\phi)=\varepsilon \triangle \phi_{t}, \quad x \in \mathbb{R}, \quad t \geq 0
$$

with initial data

$$
\phi(\cdot, 0)=\phi_{0}, \quad \phi_{t}(\cdot, 0)=\phi_{1},
$$

has a unique global solution

$$
\phi_{\varepsilon} \in C\left(\left[0,+\infty\left[; H^{3}(\mathbb{R})\right) \cap C^{1}\left(\left[0,+\infty\left[; H^{2}(\mathbb{R})\right) \cap C^{2}\left(\left[0,+\infty\left[; L^{2}(\mathbb{R})\right) .\right.\right.\right.\right.\right.\right.
$$

In this conditions, if we put $u_{\varepsilon}=\phi_{\varepsilon x}, v_{\varepsilon}=\phi_{\varepsilon t}$, we conclude that $\left(u_{\varepsilon}, v_{\varepsilon}\right) \in$ $C\left(\left[0,+\infty\left[; H^{2}(\mathbb{R})^{2}\right) \cap C^{1}\left(\left[0,+\infty\left[; L^{2}(\mathbb{R})^{2}\right)\right.\right.\right.\right.$ is the unique solution of the Cauchy problem (11), (12).

The proof that we present here generalizes to $\mathbb{R}$ the results obtained by J. Greenberg, R. Mac Camy and V. Mizel ([8]) for the viscoelasticity equations in the interval $[0,1]$, and follows these authors and J. P. Dias' ideas, who proves in [3] a result of global existence of strong solution for a similar problem in two space dimensions, considering radial symmetric initial data.

By using a classical fix point method, we begin to prove the following result of local existence: 
Theorem 2.1. Let $\phi_{0} \in H^{3}(\mathbb{R})$ and $\phi_{1} \in H^{2}(\mathbb{R})$. Then, there exists $T_{0}>0$ such that the Cauchy problem (14), (15) has a unique solution in $C\left(\left[0, T_{0}\right] ; H^{3}(\mathbb{R})\right) \cap$ $C^{1}\left(\left[0, T_{0}\right] ; H^{2}(\mathbb{R})\right) \cap C^{2}\left(\left[0, T_{0}\right] ; L^{2}(\mathbb{R})\right)$.

Proof. For simplicity, we consider $\varepsilon=1$. Let us assume that $\phi_{0} \in H^{3}(\mathbb{R}), \phi_{1} \in H^{2}(\mathbb{R})$, and let $(S(t))_{t>0}$ be the semigroup of operators of $H^{-1}(\mathbb{R})$ associated to the heat equation in $\mathbb{R}$.

We will use the following result (cf. [2], [13]):

$$
\begin{aligned}
& \text { If } \varphi \in H^{1}(\mathbb{R}) \text {, there exists } c>0 \text { such that } \\
& \qquad \phi(t)=S(t) \varphi \in C\left(\left[0,+\infty\left[; H^{1}(\mathbb{R})\right) \cap C^{1}\left(\left[0,+\infty\left[; H^{-1}(\mathbb{R})\right)\right.\right.\right.\right.
\end{aligned}
$$

satisfies

$$
\begin{aligned}
&\|\nabla \phi(t)\|_{L^{2}(\mathbb{R})} \leq \frac{c}{\sqrt{2 t}}\|\varphi\|_{L^{2}(\mathbb{R})}, \forall t>0, \\
&\|\Delta \phi(t)\|_{L^{2}(\mathbb{R})} \leq \frac{c}{\sqrt{2 t}}\|\nabla \varphi\|_{L^{2}(\mathbb{R})}, \quad \forall t>0 .
\end{aligned}
$$

Let us put, for $t>0$,

We have

$$
\widetilde{\psi}(t)=\int_{0}^{t} S(\tau) \phi_{1} d \tau+\phi_{0}
$$

$$
\begin{gathered}
\widetilde{\psi}_{t}=S(t) \phi_{1}, \quad \widetilde{\psi}_{x}=\int_{0}^{t} S(\tau) \phi_{1 x} d \tau+\phi_{0 x}, \\
\widetilde{\psi}_{x x}=\int_{0}^{t} S(\tau) \phi_{1 x x} d \tau+\phi_{0 x x}
\end{gathered}
$$

and, since $\phi_{1 x} \in H^{1}(\mathbb{R})$,

$$
\begin{aligned}
\Delta \widetilde{\psi}_{x}(t) & =\int_{0}^{t} \Delta\left(S(\tau) \phi_{1 x}\right) d \tau+\phi_{0 x x x}=\int_{0}^{t} \frac{\partial}{\partial \tau}\left(S(\tau) \phi_{1 x}\right) d \tau+\phi_{0 x x x} \\
& =S(t) \phi_{1 x}-\phi_{1 x}+\phi_{0 x x x} \quad \text { (cf. [2]). }
\end{aligned}
$$

Hence, $\widetilde{\psi} \in C\left(\left[0,+\infty\left[; H^{3}(\mathbb{R})\right), \widetilde{\psi}_{x} \in C\left(\left[0,+\infty\left[; H^{2}(\mathbb{R})\right)\right.\right.\right.\right.$ and $\widetilde{\psi}_{t} \in C([0,+\infty[;$ $\left.H^{2}(\mathbb{R})\right)$.

Let us consider, for $T>0$,

$$
X_{T}=\left\{\psi \in C\left([0, T] ; H^{3}(\mathbb{R})\right) \cap C^{1}\left([0, T] ; H^{2}(\mathbb{R})\right):\|\psi-\widetilde{\psi}\|_{X_{T}} \leq M\right\},
$$

where $\|\psi\|_{X_{T}}=\max _{[0, T]}\|\psi(t)\|_{H^{3}(\mathbb{R})}+\max _{[0, T]}\left\|\psi_{t}(t)\right\|_{H^{2}(\mathbb{R})}$ and $M$ is a positive constant such that $\|\widetilde{\psi}\| \leq M$. We will prove that there exists $T_{0}>0$ such that the problem

$$
\left\{\begin{array}{l}
\frac{\partial}{\partial t} \phi_{t}-\Delta \phi_{t}=f(\phi), \quad f(\phi)=\sigma^{\prime}\left(\phi_{x}\right) \phi_{x x}-F(\phi), \\
\phi(\cdot, 0)=\phi_{0}, \quad \phi_{t}(\cdot, 0)=\phi_{1},
\end{array}\right.
$$


has a solution $\phi \in X_{T_{0}}$.

In order to do this, we consider, for a given $\psi \in X_{T}$, the linear problem in $X_{T}$

$$
\left\{\begin{array}{l}
\frac{\partial}{\partial t} \phi_{t}-\Delta \phi_{t}=f(\psi), \\
\phi(\cdot, 0)=\phi_{0}, \phi_{t}(\cdot, 0)=\phi_{1} .
\end{array}\right.
$$

Since $(f(\psi))_{x}=\sigma^{\prime}\left(\psi_{x}\right) \psi_{x x x}+\sigma^{\prime \prime}\left(\psi_{x}\right) \psi_{x x}^{2}-F^{\prime}(\psi) \psi_{x}$, and due to the inclusion $H^{1}(\mathbb{R}) \subseteq L^{\infty}(\mathbb{R})$, we conclude that $f(\psi) \in C\left([0, T] ; H^{1}(\mathbb{R})\right)$ and so (19) has a unique solution $\phi=\mathcal{T}(\psi)$ in $[0, T]$,

$$
\phi(t)=\int_{0}^{t} \phi_{t}(\tau) d \tau+\phi_{0}
$$

where

$$
\phi_{t}(t)=S(t) \phi_{1}+\int_{0}^{t} S(t-\tau)(f(\psi))(\tau) d \tau .
$$

Next, we prove that there exists $T^{\prime}>0$ such that, for each $T<T^{\prime}, \mathcal{T}\left(X_{T}\right) \subseteq X_{T}$. Let $\psi \in X_{T}$ and $0<t \leq T$. For $\phi=\mathcal{T}(\psi)$ defined as above, we conclude from (16) and (17) that

$$
\begin{aligned}
\left\|\phi_{t}(t)-\widetilde{\psi}_{t}(t)\right\|_{H^{2}(\mathbb{R})} & =\left\|\int_{0}^{t} S(t-\tau)(f(\psi))(\tau) d \tau\right\|_{H^{2}(\mathbb{R})} \\
& \leq \int_{0}^{t} \frac{1}{\sqrt{2(t-\tau)}}\|(f(\psi))(\tau)\|_{H^{1}(\mathbb{R})} d \tau \leq g(t) C(M)
\end{aligned}
$$

and

$$
\begin{aligned}
\|\phi(t)-\widetilde{\psi}(t)\|_{H^{2}(\mathbb{R})} & =\left\|\int_{0}^{t}\left(\phi_{t}(\tau)-\widetilde{\psi}_{t}(\tau)\right) d \tau\right\|_{H^{2}(\mathbb{R})} \\
& \leq \int_{0}^{t}\left\|\phi_{t}(\tau)-\widetilde{\psi}_{t}(\tau)\right\|_{H^{2}(\mathbb{R})} d \tau \leq g(t) C(M),
\end{aligned}
$$

where $g$ is an increasing continuous function such that $g(0)=0$ and $C(M)$ is a continuous function of $\mathrm{M}$.

In order to estimate $\left\|\phi_{x}(t)-\widetilde{\psi}_{x}(t)\right\|_{H^{2}(\mathbb{R})}$, we point out that

$$
\frac{\partial}{\partial t}\left[\left(\frac{\partial}{\partial t}-\Delta\right) \phi_{x}\right]=\frac{\partial}{\partial x}\left[\left(\frac{\partial}{\partial t}-\Delta\right) \phi_{t}\right]=(f(\psi))_{x},
$$

and so

$$
\phi_{x t}-\Delta \phi_{x}=\int_{0}^{t}(f(\psi))_{x}(\tau) d \tau+\phi_{1 x}-\Delta \phi_{0 x} .
$$


As a consequence of the above considerations, we obtain

$$
\begin{aligned}
\left(\phi_{x}-\widetilde{\psi}_{x}\right)- & \Delta\left(\phi_{x}-\widetilde{\psi}_{x}\right)=\phi_{x}-\phi_{x t}+\phi_{1 x}-\Delta \phi_{0 x}+\int_{0}^{t}(f(\psi))_{x}(\tau) d \tau \\
& -\int_{0}^{t} S(\tau) \phi_{1 x} d \tau-\phi_{0 x}+S(t) \phi_{1 x}-\phi_{1 x}+\Delta \phi_{0 x} \\
= & \phi_{x}-\phi_{0 x}+S(t) \phi_{1 x}-\phi_{x t}+\int_{0}^{t}(f(\psi))_{x}(\tau) d \tau-\int_{0}^{t} S(\tau) \phi_{1 x} d \tau \\
= & \phi_{x}-\phi_{0 x}-\int_{0}^{t} S(t-\tau)(f(\psi))_{x}(\tau) d \tau \\
& +\int_{0}^{t}(f(\psi))_{x}(\tau) d \tau-\int_{0}^{t} S(\tau) \phi_{1 x} d \tau=h
\end{aligned}
$$

which allow us to conclude that $\left(\phi_{x}-\widetilde{\psi}_{x}\right)-\Delta\left(\phi_{x}-\widetilde{\psi}_{x}\right) \in L^{2}(\mathbb{R})$, because $h \in L^{2}(\mathbb{R})$, since, again by (16) and (17), we deduce that

$$
\begin{aligned}
\|\left(\phi_{x}-\right. & \left.\widetilde{\psi}_{x}\right)-\Delta\left(\phi_{x}-\widetilde{\psi}_{x}\right)\left\|_{L^{2}(\mathbb{R})}=\right\| h \|_{L^{2}(\mathbb{R})} \leq \\
\leq & \left\|\phi_{x}-\phi_{0 x}\right\|_{L^{2}(\mathbb{R})}+\int_{0}^{t}\left\|S(t-\tau)(f(\psi))_{x}(\tau)\right\|_{L^{2}(\mathbb{R})} d \tau \\
& +\int_{0}^{t}\left\|(f(\psi))_{x}(\tau)\right\|_{L^{2}(\mathbb{R})} d \tau+\int_{0}^{t}\left\|S(\tau) \phi_{1 x}\right\|_{L^{2}(\mathbb{R})} d \tau \\
\leq & g(t) C(M),
\end{aligned}
$$

because

$$
\begin{aligned}
\left\|\phi_{x}-\phi_{0 x}\right\|_{L^{2}(\mathbb{R})} & =\left\|\int_{0}^{t} \phi_{t x}(\tau) d \tau\right\|_{L^{2}(\mathbb{R})} \leq \int_{0}^{t}\left\|\phi_{t x}(\tau)\right\|_{L^{2}(\mathbb{R})} d \tau \leq \\
& \leq \int_{0}^{t}\left\|\phi_{t x}(\tau)-S(\tau) \phi_{1 x}\right\|_{L^{2}(\mathbb{R})} d \tau+\int_{0}^{t}\left\|S(\tau) \phi_{1 x}\right\|_{L^{2}(\mathbb{R})} d \tau \\
& \leq g(t) C(M)
\end{aligned}
$$

By Fourier transform we obtain

$$
\left\|\phi_{x}-\widetilde{\psi}_{x}\right\|_{H^{2}(\mathbb{R})} \leq c\left\|\left(\phi_{x}-\widetilde{\psi}_{x}\right)-\Delta\left(\phi_{x}-\widetilde{\psi}_{x}\right)\right\|_{L^{2}(\mathbb{R})} \leq g(t) C(M) .
$$

We can now choose $T^{\prime}>0$ such that $g\left(T^{\prime}\right) C(M) \leq M$ and, from (20), (21) and (23), we obtain

$$
\|\phi(t)-\widetilde{\psi}(t)\|_{H^{3}(\mathbb{R})} \leq M, \quad\left\|\phi_{t}(t)-\widetilde{\psi}_{t}(t)\right\|_{H^{2}(\mathbb{R})} \leq M,
$$

for all $0<t<T^{\prime}$. Hence, if $0<T<T^{\prime}, \mathcal{T}\left(X_{T}\right) \subseteq X_{T}$. 
Now we have that, for given $\psi, \bar{\psi} \in X_{T}\left(T<T^{\prime}\right), \phi=\mathcal{T}(\psi)$ and $\bar{\phi}=\mathcal{T}(\bar{\psi})$ satisfy

$$
\begin{aligned}
& \|\phi(t)-\bar{\phi}(t)\|_{H^{2}(\mathbb{R})}+\left\|\phi_{t}(t)-\bar{\phi}_{t}(t)\right\|_{H^{2}(\mathbb{R})} \leq \\
& \quad \leq \int_{0}^{t}\left\|\phi_{t}(\tau)-\bar{\phi}_{t}(\tau)\right\|_{H^{2}(\mathbb{R})} d \tau+\int_{0}^{t} \frac{1}{\sqrt{2(t-\tau)}}\|(f(\psi)(\tau)-f(\bar{\psi})(\tau))\|_{H^{1}(\mathbb{R})} d \tau \\
& \quad \leq g(T) C(M)\left(\max _{[0, T]}\|\psi(t)-\bar{\psi}(t)\|_{H^{3}(\mathbb{R})}+\max _{[0, T]}\left\|\psi_{t}(t)-\bar{\psi}_{t}(t)\right\|_{H^{2}(\mathbb{R})}\right) .
\end{aligned}
$$

If we proceed in the same way that we did to obtain (22), we get that

$$
\begin{aligned}
& \left\|\phi_{x}(t)-\bar{\phi}_{x}(t)\right\|_{H^{2}(\mathbb{R})} \leq \\
& g(T) C(M)\left(\max _{[0, T]}\|\psi(t)-\bar{\psi}(t)\|_{H^{3}(\mathbb{R})}+\max _{[0, T]}\left\|\psi_{t}(t)-\bar{\psi}_{t}(t)\right\|_{H^{2}(\mathbb{R})}\right) .
\end{aligned}
$$

Hence

$$
\begin{aligned}
\max _{[0, T]} \| \phi(t) & -\bar{\phi}(t)\left\|_{H^{3}(\mathbb{R})}+\max _{[0, T]}\right\| \phi_{t}(t)-\bar{\phi}_{t}(t) \|_{H^{2}(\mathbb{R})} \\
& \leq g(T) C(M)\left(\max _{[0, T]}\|\psi(t)-\bar{\psi}(t)\|_{H^{3}(\mathbb{R})}+\max _{[0, T]}\left\|\psi_{t}(t)-\bar{\psi}_{t}(t)\right\|_{H^{2}(\mathbb{R})}\right),
\end{aligned}
$$

and we can choose $T_{0}<T^{\prime}$ such that $g\left(T_{0}\right) C(M)<1$ and so $\mathcal{T}: X_{T_{0}} \longrightarrow X_{T_{0}}$ is a strict contraction in the complete normed space $X_{T_{0}}$, hence it has a unique fix point $\phi=\mathcal{T}(\phi)$, which is the unique solution of the Cauchy problem (14), (15).

Remark. Using the same notations as above, we point out that $T_{0}$ depends only on $M$ which depends only on the initial data $\phi_{0}$ and $\phi_{1}$. In consequence, since $T_{0}<T^{\prime}$, $g\left(T_{0}\right) C(M)<1$ and $g\left(T^{\prime}\right) C(M) \leq M$, we conclude that there is a minimal instant $T_{M}>0$ such that the Cauchy problem for equation (14) has solution in $\left[0, T_{M}\right]$, whatever the functions $\phi_{0}$ and $\phi_{1}$ such that $\left\|\phi_{0}\right\| \leq M,\left\|\phi_{1}\right\| \leq M$ that we consider for initial data are.

We present now the main result of this section:

Theorem 2.2. Given $\phi_{0} \in H^{3}(\mathbb{R})$ and $\phi_{1} \in H^{2}(\mathbb{R})$, the Cauchy problem (14), (15) has a unique solution in $C\left(\left[0,+\infty\left[; H^{3}(\mathbb{R})\right) \cap C^{1}\left(\left[0,+\infty\left[; H^{2}(\mathbb{R})\right) \cap C^{2}\left(\left[0,+\infty\left[; L^{2}(\mathbb{R})\right)\right.\right.\right.\right.\right.\right.$.

In order to prove this result we will obtain the following estimate for a solution $\phi$ of (14), (15):

$$
\|\phi(t)\|_{H^{3}(\mathbb{R})}+\left\|\phi_{t}(t)\right\|_{H^{2}(\mathbb{R})}+\left\|\phi_{t t}(t)\right\|_{L^{2}(\mathbb{R})} \leq c(t),
$$

where $c(t)$ is a positive continuous function.

Let $\phi \in C\left(\left[0, T\left[; H^{3}(\mathbb{R})\right) \cap C^{1}\left(\left[0, T\left[; H^{2}(\mathbb{R})\right) \cap C^{2}\left(\left[0, T\left[; L^{2}(\mathbb{R})\right)\right.\right.\right.\right.\right.\right.$ be a solution of $(14),(15)$ in $[0, T[$. 
By multiplying equation (14) by $\phi_{t}$, integrating in $\mathbb{R}$, integrating by parts and integrating in $[0, t],(0<t<T)$, we obtain

$$
\int_{\mathbb{R}}\left(\frac{\phi_{t}^{2}}{2}+\Sigma\left(\phi_{x}\right)+G(\phi)\right)(x, t) d x+\int_{0}^{t} \int_{\mathbb{R}} \phi_{t x}^{2}(x, \tau) d x d \tau=C,
$$

where $C$ depends only on the initial data $\phi_{0}$ and $\phi_{1}$.

We now assume that $\phi \in C^{2}\left(\left[0, T\left[; H^{2}(\mathbb{R})\right)\right.\right.$ (cf. [10]). By multiplying equation (14) by $\phi_{x x}$, integrating in $\mathbb{R}$ and integrating by parts, we get

$$
-\int_{\mathbb{R}} \frac{d}{d t}\left(\phi_{t x} \phi_{x}\right)+\int_{\mathbb{R}} \phi_{t x}{ }^{2}-\int_{\mathbb{R}} \sigma^{\prime}\left(\phi_{x}\right) \phi_{x x}{ }^{2}-\int_{\mathbb{R}} F^{\prime}(\phi) \phi_{x}{ }^{2}=\frac{d}{d t}\left(\int_{\mathbb{R}} \frac{\phi_{x x}{ }^{2}}{2}\right) .
$$

Integrating in $[0, t]$, we obtain

$$
\begin{aligned}
\int_{\mathbb{R}} \frac{\phi_{x x}^{2}}{2}(x, t) d x= \\
\quad-\int_{\mathbb{R}}\left(\phi_{t x} \phi_{x}\right)(x, t) d x+\int_{0}^{t} \int_{\mathbb{R}}\left(\phi_{t x}{ }^{2}-\sigma^{\prime}\left(\phi_{x}\right) \phi_{x x}{ }^{2}-F^{\prime}(\phi) \phi_{x}{ }^{2}\right) d x d \tau+C .
\end{aligned}
$$

Hence, by (26) and since $\sigma^{\prime}(u)>0, F^{\prime}(\phi) \geq 0, \forall u, \forall \phi$,

$$
\begin{aligned}
\int_{\mathbb{R}} \frac{\phi_{x x}^{2}}{2} & \leq-\int_{\mathbb{R}} \phi_{t x} \phi_{x}+C=\int_{\mathbb{R}} \phi_{t} \phi_{x x}+C \\
& \leq \int_{\mathbb{R}} \phi_{t}^{2}+\int_{\mathbb{R}} \frac{\phi_{x x}^{2}}{4}+C \leq \int_{\mathbb{R}} \frac{\phi_{x x}^{2}}{4}+C,
\end{aligned}
$$

and so

$$
\int_{\mathbb{R}} \phi_{x x}{ }^{2}(x, t) d x \leq C
$$

As we have

$$
c \int_{\mathbb{R}} \frac{\phi_{x}^{2}}{2} \leq \int_{\mathbb{R}} \Sigma\left(\phi_{x}\right),
$$

from (26) and (27) we deduce that

$$
\left\|\phi_{x}(\cdot, t)\right\|_{H^{1}(\mathbb{R})} \leq C \quad \text { and so } \quad\left\|\phi_{x}(\cdot, t)\right\|_{L^{\infty}(\mathbb{R})} \leq C .
$$

From (26) we also obtain that $\phi(t)=\int_{0}^{t} \phi_{t}(\tau) d \tau+\phi_{0}$ is such that $\|\phi\|_{L^{2} \mathbb{R}} \leq c(t)$, and then

$$
\|\phi(\cdot, t)\|_{H^{2}(\mathbb{R})} \leq c(t) .
$$


Now we derivate equation (14) in order to $t$, multiply by $\phi_{t t}$, integrate in $\mathbb{R}$ and integrate by parts. We get

$$
\frac{d}{d t}\left(\int_{\mathbb{R}} \frac{\phi_{t t}^{2}}{2}\right)+\int_{\mathbb{R}} \sigma^{\prime}\left(\phi_{x}\right) \phi_{t x} \phi_{t t x}=-\int_{\mathbb{R}} F^{\prime}(\phi) \phi_{t} \phi_{t t}-\int_{\mathbb{R}} \phi_{t t x}^{2} .
$$

By the previous estimates and from (26) and (28) we obtain

$$
\begin{aligned}
\left|\int_{\mathbb{R}} \sigma^{\prime}\left(\phi_{x}\right) \phi_{t x} \phi_{t t x}\right| & \leq \int_{\mathbb{R}}\left(\sigma^{\prime}\left(\phi_{x}\right)\right)^{2} \frac{\phi_{t x}^{2}}{2}+\int_{\mathbb{R}} \frac{\phi_{t t x}^{2}}{2} \\
& \leq c_{1} \int_{\mathbb{R}} \frac{\phi_{t x}^{2}}{2}+\int_{\mathbb{R}} \frac{\phi_{t t x}^{2}}{2}, \\
\left|\int_{\mathbb{R}} F^{\prime}(\phi) \phi_{t} \phi_{t t}\right| & \leq \int_{\mathbb{R}} \frac{\phi_{t t}^{2}}{2}+\int_{\mathbb{R}} \frac{\left(F^{\prime}(\phi)\right)^{2} \phi_{t}^{2}}{2} \\
& \leq \int_{\mathbb{R}} \frac{\phi_{t t}^{2}}{2}+c(t),
\end{aligned}
$$

and, again by (26),

$$
\frac{d}{d t} \int_{\mathbb{R}} \frac{\phi_{t t}^{2}}{2}+\int_{\mathbb{R}} \phi_{t x} \phi_{t t x} \leq c(t)+\int_{\mathbb{R}} \phi_{t t}^{2}+c_{1} \int_{\mathbb{R}} \phi_{t x}^{2} .
$$

Integrating the above inequality in $[0, t]$, we have

$$
\int_{\mathbb{R}} \frac{\phi_{t t}^{2}}{2}+\int_{\mathbb{R}} \frac{\phi_{t x}^{2}}{2} \leq c(t)+\int_{0}^{t}\left(\int_{\mathbb{R}} \frac{\phi_{t t}^{2}}{2}+c_{1} \int_{\mathbb{R}} \frac{\phi_{t x}^{2}}{2}\right),
$$

and by Gronwall's lemma we conclude that

$$
\int_{R} \frac{\phi_{t t}^{2}}{2}(x, t)+\int_{R} \frac{\phi_{t x}^{2}}{2}(x, t) \leq c(t) .
$$

Since $\phi$ is a solution of equation (14) and $|F(\phi)| \leq c_{1}|\phi|^{p}(p \geq 1)$, we have

$$
\int_{\mathbb{R}}(F(\phi))^{2} \leq c_{1}^{2} \int_{\mathbb{R}} \phi^{2 p} \leq c(t)
$$

and then

$$
\int_{\mathbb{R}} \phi_{t x x}^{2}(x, t) \leq c(t)
$$

We estimate now $\phi_{x x x}$. In order to do this, we use the following result, due to Gagliardo and Nirenberg (cf. [7]):

$$
\begin{aligned}
& \text { If } \phi \in H^{3}(\mathbb{R}) \text {, then } \phi_{x x} \in L^{4}(\mathbb{R}) \text { and } \\
& \qquad\left\|\phi_{x x}\right\|_{L^{4}(\mathbb{R})} \leq c\left\|\phi_{x x x}\right\|_{L^{2}(\mathbb{R})}{ }^{1 / 4}\left\|\phi_{x x}\right\|_{L^{2}(\mathbb{R})}{ }^{3 / 4} .
\end{aligned}
$$


If we derivate equation (14) in order to $x$, multiply by $\phi_{x x x}$, integrate in $\mathbb{R}$ and integrate by parts, we obtain

$$
\begin{aligned}
-\frac{d}{d t}\left(\int_{\mathbb{R}} \phi_{t x x} \phi_{x x}\right)+\int_{\mathbb{R}} \phi_{t x x}{ }^{2}-\int_{\mathbb{R}} \sigma^{\prime \prime}\left(\phi_{x}\right) \phi_{x x}{ }^{2} \phi_{x x x} \\
-\quad \int_{\mathbb{R}} \sigma^{\prime}\left(\phi_{x}\right) \phi_{x x x}{ }^{2}+\int_{\mathbb{R}} F^{\prime}(\phi) \phi_{x} \phi_{x x x}=\frac{d}{d t}\left(\int_{\mathbb{R}} \frac{\phi_{x x x}{ }^{2}}{2}\right),
\end{aligned}
$$

and so

$$
\begin{aligned}
\frac{d}{d t}\left(\int_{\mathbb{R}} \frac{\phi_{x x x}^{2}}{2}\right) \leq-\frac{d}{d t} & \left(\int_{\mathbb{R}} \phi_{t x x} \phi_{x x}\right) \\
& +\int_{\mathbb{R}} \phi_{t x x}{ }^{2}+\int_{\mathbb{R}} F^{\prime}(\phi) \phi_{x} \phi_{x x x}-\int_{\mathbb{R}} \sigma^{\prime \prime}\left(\phi_{x}\right) \phi_{x x}{ }^{2} \phi_{x x x} .
\end{aligned}
$$

Now, from (27) and Gagliardo-Nirenberg inequality, we have

$$
\begin{aligned}
\left|\int_{\mathbb{R}} \sigma^{\prime \prime}\left(\phi_{x}\right) \phi_{x x}{ }^{2} \phi_{x x x}\right| & \leq\left\|\sigma^{\prime \prime}\left(\phi_{x}\right)\right\|_{L^{\infty}(\mathbb{R})}\left\|\phi_{x x}\right\|_{L^{4}(\mathbb{R})}^{2}\left\|\phi_{x x x}\right\|_{L^{2}(\mathbb{R})} \\
& \leq c\left\|\phi_{x x x}\right\|_{L^{2}(\mathbb{R})}^{3 / 2}\left\|\phi_{x x}\right\|_{L^{2}(\mathbb{R})}^{3 / 2} \leq c\left(1+\left\|\phi_{x x x}\right\|_{L^{2}(\mathbb{R})}^{2}\right) .
\end{aligned}
$$

By integrating inequality $(31)$ in $[0, t]$, we obtain

$$
\int_{\mathbb{R}} \frac{\phi_{x x x}^{2}}{2}(x, t) \leq c(t) \int_{0}^{t} \int_{\mathbb{R}} \phi_{x x x}^{2}(x, \tau) d x d \tau+c(t),
$$

and, again by Gronwall's lemma,

$$
\int_{\mathbb{R}} \frac{\phi_{x x x}^{2}}{2}(x, t) \leq c(t) .
$$

From (26), (28), (29), (30) and (32) we deduce (25).

Proof of Theorem 2.2. Let $T^{*}=\sup \left\{T>0: \exists \phi \in X_{T}\right.$, solution of (14), (15) $\}$. By theorem $2.1, T^{*}>0$, and by the property of unicity we can consider a maximal solution of (14), (15),

$$
\phi \in C\left(\left[0, T^{*}\left[; H^{3}(\mathbb{R})\right) \cap C^{1}\left(\left[0, T^{*}\left[; H^{2}(\mathbb{R})\right) \cap C^{2}\left(\left[0, T^{*}\left[; L^{2}(\mathbb{R})\right) .\right.\right.\right.\right.\right.\right.
$$

If $T^{*}<+\infty$, from (25), we have that $\forall 0<t<T^{*}$,

$$
\|\phi(t)\|_{H^{3}(\mathbb{R})}+\left\|\phi_{t}(t)\right\|_{H^{2}(\mathbb{R})}+\left\|\phi_{t t}(t)\right\|_{L^{2}(\mathbb{R})} \leq c(t) \leq M^{*},
$$

where $M^{*}=\max _{\left[0, T^{*}\right]} c(t)$. According to the remark that follows the proof of Theorem 2.1, there exists $T_{M^{*}}$ such that, for all $0<t<T_{M^{*}}$, the Cauchy problem for equation (14) with initial data $\phi(\cdot, t), \phi_{t}(\cdot, t)$, has a solution in $\left[0, T_{M^{*}}\right]$. In these conditions, it is possible to extend the solution $\phi$ into a bigger time interval, which contradicts the definition of $T^{*}$. Hence, $T^{*}=+\infty$. 


\section{Young measures and reduction of their support.}

We begin this section with the following energy estimates:

Lemma 3.1. The approximated solutions $u_{\varepsilon}$ and $v_{\varepsilon}$ satisfy, for all $t>0$,

$$
\begin{gathered}
\int_{\mathbb{R}}\left(\frac{v_{\varepsilon}^{2}}{2}+\Sigma\left(u_{\varepsilon}\right)+G\left(\phi_{\varepsilon}\right)\right)(x, t) d x \leq \int_{\mathbb{R}}\left(\frac{v_{0}^{2}}{2}+\Sigma\left(u_{0}\right)+G\left(\phi_{0}\right)\right)(x) d x, \\
\varepsilon \int_{0}^{t} \int_{\mathbb{R}}\left(\sigma^{\prime}\left(u_{\varepsilon}\right) u_{\varepsilon x}{ }^{2}+v_{\varepsilon x}{ }^{2}\right)(x, \tau) d x d \tau \leq \\
3 \int_{\mathbb{R}}\left(\frac{v_{0}^{2}}{2}+\Sigma\left(u_{0}\right)+G\left(\phi_{0}\right)\right)(x) d x+\varepsilon^{2} \int_{\mathbb{R}} u_{0 x}{ }^{2}(x) d x .
\end{gathered}
$$

Proof. By multiplying the first equation of (11) by $\sigma\left(u_{\varepsilon}\right)$, the second by $v_{\varepsilon}$ and adding both equations, we obtain, since $v_{\varepsilon}=\phi_{\varepsilon t}$,

$$
\frac{d}{d t}\left(\frac{v_{\varepsilon}^{2}}{2}\right)+\frac{d}{d t}\left(\Sigma\left(u_{\varepsilon}\right)\right)-\left(\sigma^{\prime}\left(u_{\varepsilon}\right) v_{\varepsilon}\right)_{x}+\frac{d}{d t}\left(G\left(\phi_{\varepsilon}\right)\right)=\varepsilon \Delta v_{\varepsilon} v_{\varepsilon}
$$

Integrating the above equation in $\mathbb{R}$ and then by parts, we get

$$
\int_{\mathbb{R}} \frac{d}{d t}\left(\frac{v_{\varepsilon}^{2}}{2}+\Sigma\left(u_{\varepsilon}\right)+G\left(\phi_{\varepsilon}\right)\right)+\varepsilon \int_{\mathbb{R}} v_{\varepsilon x}^{2}=0
$$

If we now integrate this equation in $[0, t]$, we obtain

$$
\begin{gathered}
\int_{\mathbb{R}}\left(\frac{v_{\varepsilon}^{2}}{2}+\Sigma\left(u_{\varepsilon}\right)+G\left(\phi_{\varepsilon}\right)\right)(x, t) d x+\varepsilon \int_{0}^{t} \int_{\mathbb{R}} v_{\varepsilon x}^{2}(x, \tau) d x d \tau= \\
\int_{\mathbb{R}}\left(\frac{v_{0}^{2}}{2}+\Sigma\left(u_{0}\right)+G\left(\phi_{0}\right)\right)(x) d x
\end{gathered}
$$

and (33) follows.

In order to prove (34), we follow Serre and Shearer's ideas ([16]). Since $v_{\varepsilon x}=u_{\varepsilon t}$, we have $\Delta v_{\varepsilon}=u_{\varepsilon x t}$ and $\phi_{\varepsilon_{x x}}=u_{\varepsilon x}$. Hence, if we multiply the second equation of (11) by $u_{\varepsilon x}$ and integrate in $\mathbb{R} \times[0, t]$, we have

$$
\int_{0}^{t} \int_{\mathbb{R}}\left(u_{\varepsilon x} v_{\varepsilon t}-\sigma^{\prime}\left(u_{\varepsilon}\right) u_{\varepsilon x}{ }^{2}\right)=\int_{0}^{t} \int_{\mathbb{R}} F^{\prime}\left(\phi_{\varepsilon}\right) \phi_{\varepsilon_{x}}{ }^{2}+\varepsilon \int_{0}^{t} \int_{\mathbb{R}} \frac{d}{d t}\left(\frac{u_{\varepsilon x}{ }^{2}}{2}\right),
$$

and, since $u_{\varepsilon x t}=v_{\varepsilon x x}$, we get

$$
\int_{0}^{t} \int_{\mathbb{R}} u_{\varepsilon x} v_{\varepsilon t}=\int_{0}^{t} \int_{\mathbb{R}}\left(\left(v_{\varepsilon} u_{\varepsilon x}\right)_{t}-v_{\varepsilon} u_{\varepsilon x t}\right)=\left.\int_{\mathbb{R}} v_{\varepsilon} u_{\varepsilon x}\right|_{0} ^{t}+\int_{0}^{t} \int_{\mathbb{R}} v_{\varepsilon x}^{2},
$$


and then

$$
\left.\int_{\mathbb{R}} v_{\varepsilon} u_{\varepsilon x}\right|_{0} ^{t}+\int_{0}^{t} \int_{\mathbb{R}} v_{\varepsilon x}{ }^{2}-\left.\frac{\varepsilon}{2} \int_{\mathbb{R}} u_{\varepsilon x}{ }^{2}\right|_{0} ^{t}=\int_{0}^{t} \int_{\mathbb{R}} \sigma^{\prime}\left(u_{\varepsilon}\right) u_{\varepsilon x}{ }^{2}+\int_{0}^{t} \int_{\mathbb{R}} F^{\prime}\left(\phi_{\varepsilon}\right) \phi_{\varepsilon x}{ }^{2} .
$$

Since $F^{\prime}(\phi) \geq 0$, from the above equality we have

$$
\begin{aligned}
\int_{0}^{t} \int_{\mathbb{R}} \sigma^{\prime}\left(u_{\varepsilon}\right) u_{\varepsilon x}{ }^{2} \leq & \int_{0}^{t} \int_{\mathbb{R}} \sigma^{\prime}\left(u_{\varepsilon}\right) u_{\varepsilon x}{ }^{2}+\int_{0}^{t} \int_{\mathbb{R}} F^{\prime}\left(\phi_{\varepsilon}\right) \phi_{\varepsilon x}{ }^{2} \leq \\
& \left(\int_{\mathbb{R}} u_{\varepsilon x}{ }^{2}(t)\right)^{(1 / 2)}\left(\int_{\mathbb{R}} v_{\varepsilon}^{2}(t)\right)^{(1 / 2)}-\int_{\mathbb{R}} v_{0} u_{0 x}+\int_{0}^{t} \int_{\mathbb{R}} v_{\varepsilon x}{ }^{2}-\left.\frac{\varepsilon}{2} \int_{\mathbb{R}} u_{\varepsilon x}{ }^{2}\right|_{0} ^{t} \leq \\
& \frac{1}{2 \varepsilon} \int_{\mathbb{R}} v_{\varepsilon}{ }^{2}(t)+\frac{1}{2 \varepsilon} \int_{\mathbb{R}} v_{0}^{2}+\varepsilon \int_{\mathbb{R}} u_{0 x}{ }^{2}+\int_{0}^{t} \int_{\mathbb{R}} v_{\varepsilon x}{ }^{2} .
\end{aligned}
$$

Hence,

$$
\varepsilon \int_{0}^{t} \int_{\mathbb{R}} \sigma^{\prime}\left(u_{\varepsilon}\right) u_{\varepsilon x}{ }^{2} \leq \frac{1}{2} \int_{\mathbb{R}} v_{\varepsilon}^{2}(t)+\frac{1}{2} \int_{\mathbb{R}} v_{0}^{2}+\varepsilon^{2} \int_{\mathbb{R}} u_{0 x}{ }^{2}+\varepsilon \int_{0}^{t} \int_{\mathbb{R}} v_{\varepsilon x}{ }^{2} .
$$

The estimate (34) follows then from (35).

We now present the theorem of existence of Young measures. For the proof and more details concerning this subject, we refer to [1] and [17].

Let $\mathcal{M}(\Omega)$ be the space of finite real Radon measures on $\Omega$.

Theorem 3.2 (Young measures and representation of weak limits). Let $\eta: \mathbb{R}^{m} \longrightarrow \mathbb{R}$ be a continuous positive function such that $\frac{1}{\eta(\lambda)} \rightarrow 0,|\lambda| \rightarrow+\infty$, and $U_{\varepsilon}=\left(U_{1 \varepsilon}, \ldots, U_{m \varepsilon}\right)$ a sequence defined a. e. in $\mathbb{R} \times[0,+\infty[$ such that, for all compact set $K \subseteq \mathbb{R} \times\left[0,+\infty\left[, \exists C_{K}>0: \int_{K} \eta\left(U_{\varepsilon}(x, t)\right) d x d t \leq C_{K}\right.\right.$. Then there is a subsequence $\left(U_{\varepsilon^{\prime}}\right)_{\varepsilon^{\prime}}$ and a weakly measurable family of nonnegative measures of $\mathcal{M}\left(\mathbb{R}^{m}\right),\left\{\nu_{x, t}\right\}_{(x, t) \in \mathbb{R} \times[0,+\infty[}$, with mass equal to one a. e. $(x, t) \in \mathbb{R} \times[0,+\infty[$, such that

(i) For any continuous function $g: \mathbb{R}^{m} \longrightarrow \mathbb{R}$ such that $\frac{g(\lambda)}{\eta(\lambda)} \rightarrow 0,|\lambda| \rightarrow+\infty$, let

$$
\bar{g}(x, t)=\int_{\mathbb{R}^{m}} g(\lambda) d \nu_{x, t}(\lambda) .
$$

Then $\bar{g} \in L_{\text {loc }}^{1}\left(\mathbb{R} \times\left[0,+\infty[)\right.\right.$ and $g\left(U_{\varepsilon^{\prime}}\right) \rightarrow \bar{g}$ in the weak topology of $L_{\text {loc }}^{1}(\mathbb{R} \times$ $\left[0,+\infty[)\right.$ induced by $C_{c}(\mathbb{R} \times[0,+\infty[)$, the space of continuous functions with compact support in $\mathbb{R} \times[0,+\infty[$.

(ii) If $\frac{|\lambda|^{q}}{\eta(\lambda)} \rightarrow 0,|\lambda| \rightarrow+\infty$, and if the support of $\nu_{x, t}$ is a point a. e. $(x, t) \in \mathbb{R} \times$ $\left[0,+\infty\left[\right.\right.$, then $U_{\varepsilon^{\prime}} \rightarrow \bar{U}(x, t)=\int_{\mathbb{R}^{m}} \lambda d \nu_{x, t}(\lambda)$ in $L_{l o c}^{q}\left(\mathbb{R} \times\left[0,+\infty[), \nu_{x, t}=\delta_{\bar{U}(x, t)}\right.\right.$ and, if $g$ is in the same conditions as above, $g\left(U_{\varepsilon^{\prime}}\right) \rightarrow g(\bar{U})$ in $L_{l o c}^{1}(\mathbb{R} \times[0,+\infty[)$. 
Let $\eta(u, v)=\frac{v^{2}}{2}+\Sigma(u), \forall u, v \in \mathbb{R}$. Since the approximated solutions $u_{\varepsilon}, v_{\varepsilon}$ satisfy the energy estimate (33), for all $t>0$, we can we apply the Young measures theorem and associate to a subsequence $\left(u_{\varepsilon^{\prime}}, v_{\varepsilon^{\prime}}\right)_{\varepsilon^{\prime}}$ a family of Young measures $\left\{\nu_{x, t}\right\}_{x, t \in \mathbb{R} \times[0,+\infty[}$ that verify (i) and (ii) of theorem 3.2 .

Since

$$
\frac{u^{2}}{2}+\frac{v^{2}}{2} \leq \frac{1}{c} \Sigma(u)+\frac{v^{2}}{2},
$$

it follows from (33) that $\left(u_{\varepsilon^{\prime}}, v_{\varepsilon^{\prime}}\right)_{\varepsilon^{\prime}}$ is bounded in $L_{\text {loc }}^{2}(\mathbb{R} \times[0,+\infty[)$, and then we may consider a subsequence, which will still be called $\left(u_{\varepsilon^{\prime}}, v_{\varepsilon^{\prime}}\right)_{\varepsilon^{\prime}}$, converging weakly in $L_{\text {loc }}^{2}\left(\mathbb{R} \times\left[0,+\infty[)\right.\right.$ to functions $(u, v) \in\left(L_{l o c}^{2}\left(\mathbb{R} \times[0,+\infty[))^{2}\right.\right.$. Now, again from the above inequality, we see that, if the Young measures $\nu_{x, t}$ are Dirac measures, then, by (ii) of theorem 3.2, $\nu_{x, t}=\delta_{(u(x, t), v(x, t))}$ and $\left(u_{\varepsilon^{\prime}}, v_{\mathcal{E}^{\prime}}\right) \rightarrow(u, v)$, strongly in $L_{l o c}^{q}(\mathbb{R} \times[0,+\infty[)$, for all $q<2$.

Following [16], we state now Tartar's equation for two classes of entropy-entropy flux pairs, solutions of a Goursat problem for system (39). Since we don't have $L^{\infty}$ estimates for the approximated solutions $\left(u_{\varepsilon}, v_{\varepsilon}\right)$, we can only use the above $L^{\eta}$ Young measures and, in particular, in Tartar's equation below, we are restricted to use entropy-entropy flux pairs $(p, q)$ that verify (ii) of theorem 3.2, which means that $|p / \eta|,|q / \eta| \rightarrow 0$.

Let $(p, q)$ be an entropy-entropy flux pair. We have

$$
\left\{\begin{array}{l}
p_{u}+q_{v}=0 \\
\sigma^{\prime}(u) p_{v}+q_{u}=0 .
\end{array}\right.
$$

Since $\sigma^{\prime}(u) \geq c>0$, we can define a smooth increasing function

$$
z(u)=\int_{0}^{u} \sqrt{\sigma^{\prime}(s)} d s .
$$

We change to a Riemann coordinate system $\left(w_{1}, w_{2}\right)$ by defining

$$
w_{1}(u, v)=v+z(u), \quad w_{2}(u, v)=v-z(u) .
$$

As in [17] we also consider the change of variables $(p, q) \longrightarrow(P, Q)$, defined by

$$
\begin{gathered}
p=\frac{1}{2}\left(\sigma^{\prime}\right)^{-1 / 4}(P+Q), \\
q=\frac{1}{2}\left(\sigma^{\prime}\right)^{1 / 4}(P-Q),
\end{gathered}
$$

and rewrite equation (36) in the new coordinates:

$$
\left\{\begin{array}{l}
P_{w_{1}}=a Q, \\
Q_{w_{2}}=-a P,
\end{array}\right.
$$


where $a=a\left(w_{1}-w_{2}\right)=\sigma^{\prime \prime}\left(z^{-1}\left(\frac{w_{1}-w_{2}}{2}\right)\right) / 8\left(\sigma^{\prime}\left(z^{-1}\left(\frac{w_{1}-w_{2}}{2}\right)\right)\right)^{3 / 2}$.

We consider entropy-entropy flux pairs $(p, q)$, given by $(37),(38)$, where $P$ and $Q$ are solutions of a Goursat problem related to equation (39). The Goursat problem consists in solving system (39), with data in the lines $w_{1}=\bar{w}_{1}$ and $w_{2}=\bar{w}_{2}$, $\left(\bar{w}_{1}, \bar{w}_{2}\right) \in \mathbb{R}^{2}:$

$$
P\left(\bar{w}_{1}, w_{2}\right)=g\left(w_{2}\right), \quad Q\left(w_{1}, \bar{w}_{2}\right)=h\left(w_{1}\right) .
$$

If $g$ and $h$ are regular then the Goursat problem has a unique solution $(P, Q)$ with the same regularity and if $g$ has his support contained in the set $\left\{w_{2} \in \mathbb{R}: w_{2}>\bar{w}_{2}\right\}$, $\bar{w}_{2} \in \mathbb{R}$, then $P$ and $Q$ have their supports contained in the halfplane $\left\{\left(w_{1}, w_{2}\right) \in\right.$ $\left.\mathbb{R}^{2}: w_{2} \geq \bar{w}_{2}\right\}$. For details concerning Goursat problem we refer [14] and [16].

We now state Tartar's equation, which is deduced by applying div-curl lemma to $p_{1}\left(u_{\varepsilon}, v_{\varepsilon}\right), q_{1}\left(u_{\varepsilon}, v_{\varepsilon}\right), p_{2}\left(u_{\varepsilon}, v_{\varepsilon}\right)$ and $q_{2}\left(u_{\varepsilon}, v_{\varepsilon}\right)$, where $\left(p_{1}, q_{1}\right)$ and $\left(p_{2}, q_{2}\right)$ are entropyentropy flux pairs associated to $P$ and $Q$, solutions of a Goursat problem for the system (39) with continuous, compactly supported Goursat data, or solutions of a Cauchy problem for this system with continuous, compactly supported initial data on the line $w_{1}-w_{2}=\xi_{0}, \xi_{0}$ constant.

Let $(p, q)$ be an entropy-entropy flux pair. In order to apply div-curl lemma, we must prove that $\left(p\left(u_{\varepsilon}, v_{\varepsilon}\right)\right)_{t}+\left(q\left(u_{\varepsilon}, v_{\varepsilon}\right)\right)_{x}$ lies in a compact subset of $H_{l o c}^{-1}(\mathbb{R} \times$ $\left[0,+\infty[)\right.$. Multiplying system $(11)$ by $\left(p_{u}\left(u_{\varepsilon}, v_{\varepsilon}\right), p_{v}\left(u_{\varepsilon}, v_{\varepsilon}\right)\right)$, we obtain

$$
\begin{aligned}
\left(p\left(u_{\varepsilon}, v_{\varepsilon}\right)\right)_{t}+\left(q\left(u_{\varepsilon}, v_{\varepsilon}\right)\right)_{x} & =\varepsilon p_{v} v_{\varepsilon x x}-p_{v} F\left(\phi_{\varepsilon}\right) \\
& =\varepsilon\left(p_{v} v_{\varepsilon x}\right)_{x}-\varepsilon\left(p_{u v} u_{\varepsilon x} v_{\varepsilon x}+p_{v v} v_{\varepsilon x}{ }^{2}\right)-p_{v} F\left(\phi_{\varepsilon}\right),
\end{aligned}
$$

where, in the second member, the derivatives refer to the point $\left(u_{\varepsilon}, v_{\varepsilon}\right)$.

To use Murat's lemma (cf. [6]) we need to have the following conditions:

M1 $\left(p\left(u_{\varepsilon}, v_{\varepsilon}\right)+q\left(u_{\varepsilon}, v_{\varepsilon}\right)\right)_{\varepsilon}$ is uniformly bounded in $L_{l o c}^{p}(\mathbb{R} \times[0,+\infty[)$, for some $p>$ 2

M2 $\left(\varepsilon\left(p_{v} v_{\varepsilon x}\right)_{x}\right)_{\varepsilon}$ is precompact in $H_{l o c}^{-1}(\mathbb{R} \times[0,+\infty[)$;

M3 $\left(\varepsilon\left(p_{u v} u_{\varepsilon x} v_{\varepsilon x}+p_{v v} v_{\varepsilon x}{ }^{2}\right)\right)_{\varepsilon}$ is uniformly bounded in $L_{l o c}^{1}(\mathbb{R} \times[0,+\infty[)$;

M4 $\left(p_{v} F\left(\phi_{\varepsilon}\right)\right)_{\varepsilon}$ is uniformly bounded in $L_{l o c}^{1}(\mathbb{R} \times[0,+\infty[)$.

We remark that, if M1 holds, then $\left(\left(p\left(u_{\varepsilon}, v_{\varepsilon}\right)\right)_{t}+\left(q\left(u_{\varepsilon}, v_{\varepsilon}\right)\right)_{x}\right)_{\varepsilon}$ is uniformly bounded in $W_{l o c}^{-1, p}\left(\mathbb{R} \times\left[0,+\infty[)\right.\right.$, and, in M3 and M4, the bound in $L_{l o c}^{1}(\mathbb{R} \times[0,+\infty[)$ implies a bound in $\mathcal{M}(\omega)$, for any open bounded set $\omega$ of $\mathbb{R} \times[0,+\infty[$. Then, if M1-M4 hold, we can apply Murat's lemma to $\left(\left(p\left(u_{\varepsilon}, v_{\varepsilon}\right)\right)_{t}+\left(q\left(u_{\varepsilon}, v_{\varepsilon}\right)\right)_{x}\right)_{\varepsilon}$.

Theorem 3.3 (Tartar's equation). Let $\left(p_{1}, q_{1}\right)$ and $\left(p_{2}, q_{2}\right)$ be entropy-entropy flux pairs, given by (37), (38), where $P_{1}, Q_{1}, P_{2}$ and $Q_{2}$ are either solutions of a Goursat problem for system (39), with continuous, compactly supported Goursat data, or are solutions of a Cauchy problem for the same system, with continuous, 
compactly supported initial data on the line $w_{1}-w_{2}=\xi_{0}, \xi_{0}$ constant. Then $p_{1}, q_{1}$, $p_{2}$ and $q_{2}$ satisfy Tartar's equation

$$
<\nu, p_{1} q_{2}-p_{2} q_{1}>=<\nu, p_{1}><\nu, q_{2}>-<\nu, p_{2}><\nu, q_{1}>,
$$

where $\nu=\nu_{x, t}$ is the Young measure associated to the subsequence $\left(u_{\varepsilon^{\prime}}, v_{\varepsilon^{\prime}}\right)_{\varepsilon^{\prime}}$ of the approximated solutions, and $\langle\nu, p\rangle=\int p(\lambda) d \nu(\lambda)$.

Proof. In the case of entropy-entropy flux pairs solutions of the Goursat problem, we have to prove M1-M4 and apply Murat's lemma and then div-curl lemma. The proof of M1-M3 is the same as in [17]. To obtain M4 we consider a compact set $K \subseteq \mathbb{R} \times[0,+\infty[$. If $t>0$, we have

$$
\begin{aligned}
\left\|\phi_{\varepsilon}(\cdot, t)\right\|_{L^{2}(\mathbb{R})}^{2} & =\int_{\mathbb{R}} \phi_{\varepsilon}{ }^{2}(x, t) d x \leq C \int_{\mathbb{R}}\left(\int_{0}^{t} v_{\varepsilon}(x, \tau) d \tau\right)^{2}+\phi_{0}{ }^{2} d x \\
& \leq C\left\|\phi_{0}\right\|_{L^{2}(\mathbb{R})}^{2}+C \int_{\mathbb{R}} \int_{0}^{t} v_{\varepsilon}^{2}(x, \tau) d x d \tau \\
& \leq C+c(t) \sup _{[0, t]}\left\|v_{\varepsilon}(\cdot, \tau)\right\|_{L^{2}(\mathbb{R})} .
\end{aligned}
$$

Then, from (33) follows that $\left\|\phi_{\varepsilon}(\cdot, t)\right\|_{L^{2}(\mathbb{R})} \leq c(t)$, where $c$ is a continuous function. Since $\phi_{\varepsilon_{x}}=u_{\varepsilon}$, we also obtain from this estimate that $\left\|\phi_{\varepsilon_{x}}(\cdot, t)\right\|_{L^{2}(\mathbb{R})} \leq c$. Then we have $\left\|\phi_{\varepsilon}(\cdot, t)\right\|_{H^{1}(\mathbb{R})} \leq c(t)$ and $\left\|\phi_{\varepsilon}\right\|_{L^{\infty}(K)} \leq c(t)$. Since $F$ is continuous, we have

$$
\int_{K}\left|F\left(\phi_{\varepsilon}\right)\right| d x d t \leq C,
$$

hence $\left(F\left(\phi_{\varepsilon}\right)\right)_{\varepsilon}$ is uniformly bounded in $L_{l o c}^{1}\left(\mathbb{R} \times\left[0,+\infty[)\right.\right.$, and, since $\left(p_{v}\left(u_{\varepsilon}, v_{\varepsilon}\right)\right)_{\varepsilon}$ is uniformly bounded in $L^{\infty}(\mathbb{R} \times[0,+\infty[)$ (cf. [17]), we obtain M4.

If M1-M4 hold, then Murat's lemma and div-curl lemma imply Tartar's equation.

To prove the case where $P$ and $Q$ are solutions of the Cauchy problem, we refer to $[16]$.

Now, as in [17] for the case where $\sigma^{\prime \prime}$ is never null, or as in [16] for the case where $\sigma^{\prime \prime}$ is null only once, we have the following result:

Theorem 3.4 (Reduction of the support of $\boldsymbol{\nu}$ ). The Young measure $\nu_{x, t}$ is a point mass.

For the proof, see the references indicated above.

\section{Convergence of the approximated solutions; Proof of theo- rem 1.1.}

Let $\left(u_{\varepsilon}, v_{\varepsilon}\right) \in C\left(\left[0,+\infty\left[; H^{2}(\mathbb{R})^{2}\right) \cap C^{1}\left(\left[0,+\infty\left[; L^{2}(\mathbb{R})^{2}\right)\right.\right.\right.\right.$ be the solution of the Cauchy problem for the approximated system (11), with initial data (12). 
Let us consider $\varphi$ and $\psi \in C_{0}^{\infty}(\mathbb{R} \times[0,+\infty[)$. By multiplying the first equation of the system (11) by $\varphi$, the second by $\psi$, adding the resulting equations and integrating by parts in $\mathbb{R} \times\left[0,+\infty\right.$ [, we obtain that $u_{\varepsilon}$ and $v_{\varepsilon}$ satisfy the weak formulation of the Cauchy problem (11), (12),

$$
\begin{aligned}
\int_{\mathbb{R}} \int_{0}^{+\infty}\left(u_{\varepsilon} \varphi_{t}-v_{\varepsilon} \varphi_{x}\right) d x d t+\int_{\mathbb{R}} u_{0} \varphi(x, 0) d x+ & \\
\int_{\mathbb{R}} \int_{0}^{+\infty}\left(v_{\varepsilon} \psi_{t}-\sigma\left(u_{\varepsilon}\right) \psi_{x}-F\left(\phi_{\varepsilon}\right) \psi\right) d x d t & +\int_{\mathbb{R}} v_{0} \psi(x, 0) d x= \\
& -\varepsilon \int_{\mathbb{R}} \int_{0}^{+\infty} v_{\varepsilon} \psi_{x x} d x d t .
\end{aligned}
$$

We want to pass to the limit the above equation.

From the previous section we have that the support of the Young measures $\nu_{x, t}$ is reduced to a point. Let, for $(x, t) \in \mathbb{R} \times[0,+\infty[,(\bar{u}(x, t), \bar{v}(x, t))$ be the support of the Young measure $\nu_{x, t}$. Let $p<2$. Since

we have

$$
\eta(u, v) \geq c \frac{v^{2}}{2}+\frac{u^{2}}{2}
$$

$$
0 \leq \frac{|u|^{p}+|v|^{p}}{\eta(u, v)} \leq C \frac{|u|^{p}+|v|^{p}}{v^{2}+u^{2}} \rightarrow 0, \quad|u|+|v| \rightarrow+\infty .
$$

Then, from property (ii) of the Young measures theorem, we have

$$
\bar{u}(x, t)=\int_{\mathbb{R}^{2}} \lambda_{1} d \nu_{x, t}\left(\lambda_{1}, \lambda_{2}\right), \quad \bar{v}(x, t)=\int_{\mathbb{R}^{2}} \lambda_{2} d \nu_{x, t}\left(\lambda_{1}, \lambda_{2}\right) \in L_{l o c}^{p}(\mathbb{R} \times[0,+\infty[)
$$

and $\left(u_{\varepsilon^{\prime}}, v_{\varepsilon^{\prime}}\right) \rightarrow(\bar{u}, \bar{v})$, strongly in $\left(L_{l o c}^{p}\left(\mathbb{R} \times[0,+\infty[))^{2}\right.\right.$. We had previously seen that a subsequence $\left(u_{\varepsilon^{\prime}}, v_{\varepsilon^{\prime}}\right)_{\varepsilon^{\prime}}$ converged, weakly in $\left(L_{\text {loc }}^{2}\left(\mathbb{R} \times[0,+\infty[))^{2}\right.\right.$, to a function $(u, v) \in L_{l o c}^{2}\left(\mathbb{R} \times\left[0,+\infty[)^{2}\right.\right.$, and so, by the unicity of weak limit, we may conclude that $(\bar{u}, \bar{v})=(u, v) \in\left(L_{l o c}^{2}\left(\mathbb{R} \times[0,+\infty[))^{2}\right.\right.$.

Since $\sigma$ satisfies $\mathrm{H} 4$, we have

$$
\frac{\sigma(u)}{\eta(u, v)} \rightarrow 0, \quad \text { if }|u|+|v| \rightarrow+\infty,
$$

and again from (ii) we conclude that $\sigma\left(u_{\varepsilon^{\prime}}\right) \rightarrow \sigma(\bar{u})$ in $L_{l o c}^{1}(\mathbb{R} \times[0,+\infty[)$.

Due to what was exposed above, it follows immediately that

$$
\begin{aligned}
\lim _{\varepsilon^{\prime} \rightarrow 0} \int_{\mathbb{R}} \int_{0}^{+\infty}\left(u_{\varepsilon^{\prime}} \varphi_{t}-v_{\varepsilon^{\prime}} \varphi_{x}\right) d x d t & =\int_{\mathbb{R}} \int_{0}^{+\infty}\left(\bar{u} \varphi_{t}-\bar{v} \varphi_{x}\right) d x d t, \\
\lim _{\varepsilon^{\prime} \rightarrow 0} \int_{\mathbb{R}} \int_{0}^{+\infty} v_{\varepsilon^{\prime}} \psi_{t} d x d t & =\int_{\mathbb{R}} \int_{0}^{+\infty} \bar{v} \psi_{t} d x d t \\
\lim _{\varepsilon^{\prime} \rightarrow 0} \int_{\mathbb{R}} \int_{0}^{+\infty} \sigma\left(u_{\varepsilon^{\prime}}\right) \psi_{x} d x d t & =\int_{\mathbb{R}} \int_{0}^{+\infty} \sigma(\bar{u}) \psi_{x} d x d t
\end{aligned}
$$


Now, since

$$
\begin{aligned}
\left|\varepsilon^{\prime} \int_{\mathbb{R}} \int_{0}^{+\infty} v_{\varepsilon^{\prime}} \psi_{x x} d x d t\right| & \leq\left\|\psi_{x x}\right\|_{L^{\infty}} \varepsilon^{\prime} \int_{\sup (\psi)}\left|v_{\varepsilon^{\prime}}\right| d x d t \\
& \leq\left\|\psi_{x x}\right\|_{L^{\infty}}(\operatorname{m}(\sup (\psi)))^{1 / 2} \varepsilon^{\prime}\left(\int_{\sup (\psi)} v_{\varepsilon^{\prime}}^{2} d x d t\right)^{1 / 2},
\end{aligned}
$$

we obtain, provided that, as a consequence of $(33),\left(v_{\varepsilon^{\prime}}\right)_{\varepsilon^{\prime}}$ is uniformly bounded in $L^{2}(\sup (\psi))$,

$$
\lim _{\varepsilon^{\prime} \rightarrow 0} \varepsilon^{\prime} \int_{\mathbb{R}} \int_{0}^{+\infty} v_{\varepsilon^{\prime}} \psi_{x x} d x d t=0 .
$$

To show that $(\bar{u}, \bar{v})$ is a weak solution of the problem $(4),(5)$, we now study the limit of

$$
\int_{\mathbb{R}} \int_{0}^{+\infty} F\left(\phi_{\varepsilon^{\prime}}\right) \psi d x d t
$$

Let $\phi=\int_{0}^{t} \bar{v}(x, \tau) d \tau+\phi_{0}$ and $K \subseteq[a, b] \times[0, T]$ be a compact set of $\mathbb{R} \times[0,+\infty[$.

$$
\begin{aligned}
\left|\int_{K} \phi_{\varepsilon^{\prime}}(x, t)-\phi(x, t) d x d t\right| & =\left|\int_{K} \int_{0}^{t} v_{\varepsilon^{\prime}}(x, \tau)-\bar{v}(x, \tau) d \tau d x d t\right| \\
& \leq \int_{a}^{b} \int_{0}^{T} \int_{0}^{T}\left|v_{\varepsilon^{\prime}}(x, \tau)-\bar{v}(x, \tau)\right| d \tau d x d t \\
& =T \int_{a}^{b} \int_{0}^{T}\left|v_{\varepsilon^{\prime}}(x, \tau)-\bar{v}(x, \tau)\right| d x d \tau \\
& \leq T T^{1 / q}(b-a)^{1 / q}\left\|v_{\varepsilon^{\prime}}-\bar{v}\right\|_{L^{p}([a, b] \times[0, T])} \rightarrow 0,
\end{aligned}
$$

and so $\phi_{\varepsilon^{\prime}} \rightarrow \phi$ in $L_{l o c}^{1}(\mathbb{R} \times[0,+\infty[)$. Hence, there exists a subsequence, that we still call $\phi_{\varepsilon^{\prime}}$, which converges pointwise, a. e. $(x, t) \in \mathbb{R} \times[0,+\infty[$, to $\phi$. Since $F$ is continuous, $F\left(\phi_{\varepsilon^{\prime}}(x, t)\right) \rightarrow F(\phi(x, t))$, a. e. $(x, t) \in \mathbb{R} \times[0,+\infty[$.

On the other hand, for $t>0$, we show, as we did to obtain property M4 in section 3 , that

$$
\left\|\phi_{\varepsilon^{\prime}}(\cdot, t)\right\|_{L^{2}(\mathbb{R})} \leq c(t), \quad\left\|\phi_{\varepsilon^{\prime} x}(\cdot, t)\right\|_{L^{2}(\mathbb{R})} \leq c,
$$

which implies that $\left\|\phi_{\varepsilon^{\prime}}(\cdot, t)\right\|_{H^{1}(\mathbb{R})} \leq c(t)$ and $\left\|\phi_{\varepsilon^{\prime}}\right\|_{L^{\infty}(\mathbb{R} \times[0, t])} \leq c(t)$.

Now, we can apply dominated convergence theorem to (45) to obtain

$$
\lim _{\varepsilon^{\prime} \rightarrow 0} \int_{\mathbb{R}} \int_{0}^{+\infty} F\left(\phi_{\varepsilon^{\prime}}\right) \psi d x d t=\int_{\mathbb{R}} \int_{0}^{+\infty} F(\phi) \psi d x d t .
$$

From (41), (42), (43), (44) and (46) we have that $\bar{u}$ and $\bar{v}$ satisfy the weak formulation of the Cauchy problem (4), (5), and, from (33), it follows that $u_{\varepsilon}$ and $v_{\varepsilon}$ also 
satisfy

$$
\int_{\mathbb{R}}\left(\frac{v_{\varepsilon}^{2}}{2}+\Sigma\left(u_{\varepsilon}\right)\right)(x, t) \leq C, \quad \forall t>0 .
$$

By passing the above inequality to the limit, we obtain

$$
\int_{\mathbb{R}}\left(\frac{\bar{v}^{2}}{2}+\Sigma(\bar{u})\right)(x, t) \leq C, \quad \forall t>0,
$$

and so $(\bar{u}, \bar{v}) \in L^{\infty}\left(\left[0,+\infty\left[; L^{\eta}\right)\right.\right.$ is a weak solution of the Cauchy problem (4), (5).

To complete the proof of theorem 1.1, we show that the entropy inequality (9) is satisfied by the entropy-entropy flux pair defined by (10).

Since $\nabla p(u, v) \cdot \nabla f(u, v)=\nabla q(u, v), \forall(u, v) \in \mathbb{R}^{2},(f(u, v)=(-v,-\sigma(u)))$, if we multiply system $(11)$ by $(\nabla p)\left(u_{\varepsilon}, v_{\varepsilon}\right)=\left(p_{u}\left(u_{\varepsilon}, v_{\varepsilon}\right), p_{v}\left(u_{\varepsilon}, v_{\varepsilon}\right)\right)$, since $p_{u v}=0$, we conclude that

$$
\begin{aligned}
& p\left(u_{\varepsilon}, v_{\varepsilon}\right)_{t}+q\left(u_{\varepsilon}, v_{\varepsilon}\right)_{x}+\nabla p\left(u_{\varepsilon}, v_{\varepsilon}\right) \cdot(0, F(\phi))= \\
& \quad \varepsilon\left(p_{v}\left(u_{\varepsilon}, v_{\varepsilon}\right) v_{\varepsilon x}\right)_{x}-\varepsilon\left(p_{v}\left(u_{\varepsilon}, v_{\varepsilon}\right)\right)_{x} v_{\varepsilon x}=\varepsilon\left(p_{v}\left(u_{\varepsilon}, v_{\varepsilon}\right) v_{\varepsilon x}\right)_{x}-\varepsilon p_{v v}\left(u_{\varepsilon}, v_{\varepsilon}\right) v_{\varepsilon x}{ }^{2}
\end{aligned}
$$

Since the second derivative in the equation above is positive, we have that, for $\psi \in \mathcal{D}(\mathbb{R} \times] 0,+\infty[), \psi \geq 0$,

$$
\begin{aligned}
\int_{\mathbb{R}} \int_{0}^{+\infty}\left(p\left(u_{\varepsilon}, v_{\varepsilon}\right) \psi_{t}+q\left(u_{\varepsilon}, v_{\varepsilon}\right) \psi_{x}-p_{v}\left(u_{\varepsilon}, v_{\varepsilon}\right) F\left(\phi_{\varepsilon}\right) \psi\right) d x d t & \\
& -\varepsilon \int_{\mathbb{R}} \int_{0}^{+\infty}\left(p_{v}\left(u_{\varepsilon}, v_{\varepsilon}\right)\right) v_{\varepsilon x} \psi_{x} \geq 0 .
\end{aligned}
$$

Now, $p_{v}\left(u_{\varepsilon}, v_{\varepsilon}\right)=v_{\varepsilon}$ and $\varepsilon\left|v_{\varepsilon} v_{\varepsilon x}\right|=\varepsilon^{1 / 2} \varepsilon^{1 / 2}\left|v_{\varepsilon} v_{\varepsilon x}\right| \leq \varepsilon^{1 / 2}\left(\frac{v_{\varepsilon}^{2}}{2}+\frac{\varepsilon v_{\varepsilon x}^{2}}{2}\right)$. Hence, from (33) and (34) follows that the second term in the above inequality converges to 0 . Since $p$ and $q$ are continuous, by passing both members of the above inequality to the limit, we obtain

$$
\int_{\mathbb{R}} \int_{0}^{+\infty}\left(p(\bar{u}, \bar{v}) \psi_{t}+q(\bar{u}, \bar{v}) \psi_{x}-p_{v}(\bar{u}, \bar{v}) F(\phi) \psi\right) d x d t \geq 0 .
$$

This finishes the proof of theorem 1.1.

\section{References}

[1] J. M. Ball, A version of the fundamental theorem for Young measures, PDEs and Continuum Models of Phase Transitions (Nice, 1988), Lecture Notes in Phys., vol. 344, Springer, Berlin, 1989, pp. 207-215. 
[2] H. Brézis and T. Cazenave, Linear semigroups of contractions, Publications du Laboratoire d'Analyse Numérique, notes du cours de DEA 1992-1993.

[3] J.-P. Dias, On the existence of a global strong radial symmetric solution for a third-order nonlinear evolution equation in two space dimensions, J. Math. Pures Appl. (9) 80 (2001), 535546.

[4] J.-P. Dias and M. Figueira, Existence d'une solution faible pour une équation d'ondes quasilinéaire avec un terme de source semi-linéaire, C. R. Acad. Sci. Paris Sér. I Math. 322 (1996), 619-624.

[5] R. J. DiPerna, Convergence of approximate solutions to conservation laws, Arch. Rational Mech. Anal. 82 (1983), 27-70.

[6] L. C. Evans, Weak convergence methods for nonlinear partial differential equations, CBMS Regional Conference Series in Mathematics, vol. 74, Published for the Conference Board of the Mathematical Sciences, Washington, DC, 1990, ISBN 0-8218-0724-2.

[7] A. Friedman, Partial differential equations, Holt, Rinehart and Winston, Inc., New York, 1969.

[8] J. M. Greenberg, R. C. MacCamy, and V. J. Mizel, On the existence, uniqueness, and stability of solutions of the equation $\sigma^{\prime}\left(u_{x}\right) u_{x x}+\lambda u_{x t x}=\rho_{0} u_{t t}$, J. Math. Mech. 17 (1967/1968), 707728 .

[9] P. Marcati and R. Natalini, Global weak entropy solutions to quasilinear wave equations of Klein-Gordon and sine-Gordon type, J. Math. Soc. Japan 50 (1998), 433-449.

[10] A. Matsumura, Global existence and asymptotics of the solutions of the second-order quasilinear hyperbolic equations with the first-order dissipation, Publ. Res. Inst. Math. Sci. 13 (1977/78), 349-379.

[11] F. Murat, Compacité par compensation, Ann. Scuola Norm. Sup. Pisa Cl. Sci. (4) 5 (1978), 489-507.

[12] A. Pazy, Semigroups of linear operators and applications to partial differential equations, Applied Mathematical Sciences, vol. 44, Springer-Verlag, New York, 1983, ISBN 0-387-90845-5.

[13] J. Rauch, Partial differential equations, Graduate Texts in Mathematics, vol. 128, SpringerVerlag, New York, 1991, ISBN 0-387-97472-5.

[14] D. Serre, Systèmes de lois de conservation. II, Fondations. [Foundations], Diderot Editeur, Paris, 1996, ISBN 2-84134-072-4.

[15] __ La compacité par compensation pour les systèmes hyperboliques non linéaires de deux équations à une dimension d'espace, J. Math. Pures Appl. (9) 65 (1986), 423-468.

[16] D. Serre and J. Shearer, Convergence with physical viscosity for nonlinear elasticity, Lyon, 1993 (preprint).

[17] J. W. Shearer, Global existence and compactness in $L^{p}$ for the quasi-linear wave equation, Comm. Partial Differential Equations 19 (1994), 1829-1877.

[18] L. Tartar, Compensated compactness and applications to partial differential equations, Nonlinear Analysis and Mechanics: Heriot-Watt Symposium, Vol. IV, Res. Notes in Math., vol. 39, Pitman, Boston, Mass., 1979, pp. 136-212. 